\title{
A Research and Application of the Processing Methods of Railway Measurement Based on Self-Mobile Scanning Measurement System
}

\author{
Jiancai Zhang ${ }^{\mathrm{a}, \mathrm{c}, \mathrm{d}}$, Hang Mu ${ }^{\mathrm{b}, 1}$, Feng Han ${ }^{\mathrm{b}}$, Shumin Han ${ }^{\mathrm{a}, \mathrm{c}, \mathrm{d}}$ \\ ${ }^{a}$ Faculty of Geomatics, Lanzhou Jiaotong University, Lanzhou 730070, China; \\ ${ }^{b}$ College of Civil Engineering, Lanzhou Jiaotong University, Lanzhou 730070, China; \\ ${ }^{\mathrm{c}}$ National-Local Joint Engineering Research Center of Technologies and Applications \\ for National Geographic State Monitoring, Lanzhou 730070, China; \\ ${ }^{\mathrm{d}}$ Gansu Provincial Engineering Laboratory for National Geographic State Monitoring, \\ Lanzhou 730070, China
}

\begin{abstract}
With the gradual improvement of China's railway net, the opening of international railways as well as the continuous growth of railway operating mileage, the workload of remeasuring railways is increasing. The traditional methods of remeasuring railways can not meet current high-speed and highdensity operating conditions anymore in terms of safety, efficiency and quality, so a safer and more efficient measurement method is urgently needed.This thesis integrated various sensors on a self-mobile instrument, such as 3D laser scanner, digital image sensor and GNSS_IMU, designing a set of intelligent and integrated self-mobile scanning measurement system. This thesis proposed region growing segmentation based on the reflection intensity of point cloud. Through the secondary development of $\mathrm{CAD}$, the menu for automatic processing of self-mobile scanning measurement system is designed to realize rail automatic segmentation, extraction of rail top points, fitting of plane parameters of railway line, calculation of curve elements and mileage management.The results show that self-mobile scanning measurement system overcomes the shortcomings of traditional railway measurement to some extent, and realizes intelligent measurement of railways.
\end{abstract}

Keywords. Self-mobile scanning measurement system, region growing segmentation, intelligent measurement

\section{Introduction}

Remeasuring railways is an important part of railway operation and maintenance, extension of station tracks, and reconstruction of the railways, which works from various aspects, such as the elevation measurement of rail surface, equipment annotation, mileage measurement, cross-sections measurement, and topographical map measurement [1,2]. The traditional railway measurement adopts the operation mode of manual measurement, using steel ruler, total station instrument, theodolite, and leveling

${ }^{1}$ Corresponding Author, Hang Mu, Qin'an, Gansu, Doctoral candidate, lecturer. E-mail : 416958890@qq.com. 
instrument as well as GPS-RTK. In recent years, many scholars have tried to apply the 3D laser scanner and airborne lidar to railway measurement. The measuring accuracy of 3D laser scanner is high, but in the process of measurement, it is necessary to select appropriate position of $3 \mathrm{D}$ laser scanner and target ball position for station change measurement in the measurement process, which can not complete the measurement of the whole railway in a short closing time. Although airborne lidar can complete the acquisition of measurement data in an efficient way, it is limited by airspace control or blocked by the object on the ground, and can not complete the task of measuring in culvert and tunnel, so it only stays in the exploration stage.

With the opening and operation of plateau railways and alpine railways and the gradual realization of the strategic goal of "going out" for high-speed railways, the traditional manual measurement of railways is faced with the challenges of harsh environment, such as ultra-low temperature, long distance, and high altitude. It is urgent to change the method of railway measurement. Self-mobile scanning measurement system is undoubtedly the best choice, which can replace manual measurement of remeasuring railways under extreme environmental conditions, ensure the safety of workers and equipment, improve efficiency and in line with the principle of people-oriented, which is of great significance to the sustainable development of railway industry $[3,4]$.

\section{Data Acquisition}

The self-mobile scanning measurement system (see Figure 1) is an active and dynamic measurement system which integrates Leica PegasusTwo Ultimate mobile scanning system on a self-mobile instrument designed by the team, which can acquire the 3D point cloud data (see Figure 2) and panoramic images of railways (see Figure 3) and their surrounding terrain in real time. Leica PegasusTwo Ultimate mobile scanning system is composed of various sensors, such as 3D laser scanner, digital image sensor, global navigation satellite system (GNSS) and inertial measurement unit (IMU).

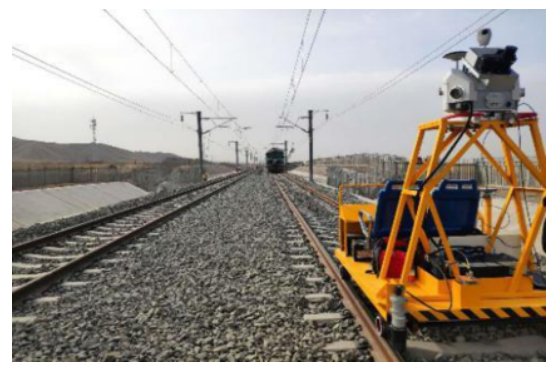

Figure 1. The self-mobile scanning measurement system.

Before data acquisition, some GNSS static base stations are set up every 20 kilometers along the railway, and are turned on one hour in advance to synchronize the GNSS static base stations with the position system of automatic scanning measurement system so as to ensure the stability and continuity of GNSS signal. CPIII points are used as control points to ensure the accuracy of point cloud, and checkpoints are set up with certain distance to ensure the correctness of data. When measuring, the instrument should keep moving at a constant speed, with the maximum speed of $20 \mathrm{~km} / \mathrm{h}$. It can 
collect 1 million points in a second and the ranging accuracy can be up to $1 \mathrm{~mm}$, what's more, the absolute accuracy of plane and elevation can be up to $2 \mathrm{~cm}$ when there is no control point in open environment. When CPIII points are used as the control point, the plane precision of point cloud can be up to $8 \mathrm{~mm}$ and the accuracy of elevation can be 4 $\mathrm{mm}$. High-precision point cloud data and high-definition panoramic images can be obtained at the same time. It can restore the status of railway, and provide location information and image data for the construction of smart railways.

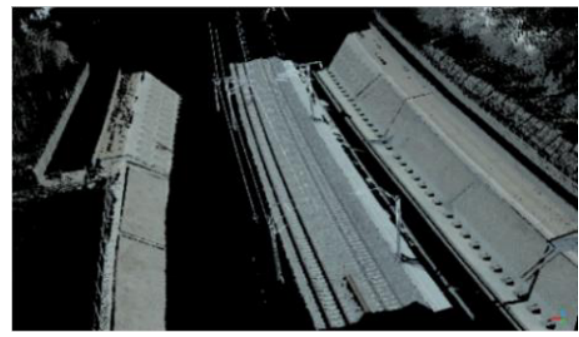

Figure 2. Point cloud data of railway

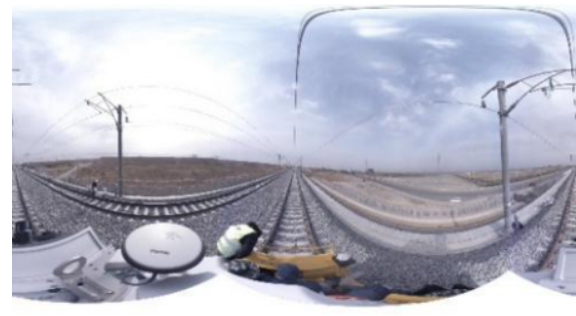

Figure 3. Panoramic images and of railway

\section{Point Cloud Data Processing}

\subsection{Point Cloud Data Preprocessing}

LIDAR point cloud data has the characteristics of irregular distribution, discontinuous coordinates and large amount of data. Due to the sensors can not move forward uniformly in the measurement process, so the resolution of the point cloud data that acquired in the measurement process is also different. In order to facilitate the subsequent processing of point cloud data, it is necessary to preprocess the original point cloud data after exporting the original point cloud. Preprocessing point cloud data mainly includes filtering and simplification $[5,6]$.

The simplification of point cloud includes removing invalid point cloud and subsampling point cloud data. Firstly, the invalid point cloud data of object on the ground is manually deleted, and then the point cloud is subsampled by using VoxelGrid subsampling. Voxel is a small cube with the side length " $L$ ". Set reasonable side length of voxel to divide the point cloud data, and use the center of gravity of all points in voxel to approximately display other points in voxel to reach the purpose of simplification of point cloud [7].

When acquiring data, noise points and outliers inevitably appear in the original point cloud data because of the influence of equipment, operators, environment and other factors, which directly affects the accuracy and smoothness of the point cloud data. Through filtering, it can effectively reduce the deviation value of the original point cloud data at these deviation points [8]. Because the average distance between outliers, noise points and main points is large, the average distance among main points is small. This thesis uses statistical analysis filtering algorithm to remove noise points and outlier points, supposing the total number of point cloud is $n$. After inputting the data of point cloud, define the number $k$ of neighbor points that are among the point $P_{0}$. First calculate the distance $d$ between the point $P_{0}$ and its neighbor points, then calculate the average value to obtain the mean value $d_{0}$ of distance of point $P_{0}$. Second 
calculate the average distance $d_{i}$ of all the points in turn, and calculate the average value of average distance, that is the global average distance $D$. Finally calculate the standard deviation $\sigma$, and set the distance threshold $M$. For all points, the average distance $d_{i}$ and the distance threshold $M$ of each point are compared. The outlier and noise point are the ones whose average distance $d_{i}$ is greater than the distance threshold $M$, otherwise it is the inner point. The pictures of point cloud data before and after preprocessing is shown in Figure 4.

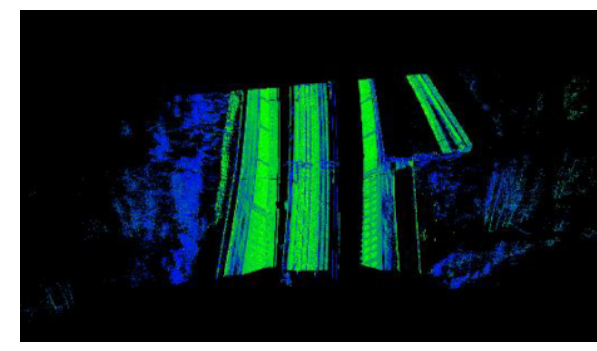

a. Before preprocessing point cloud data

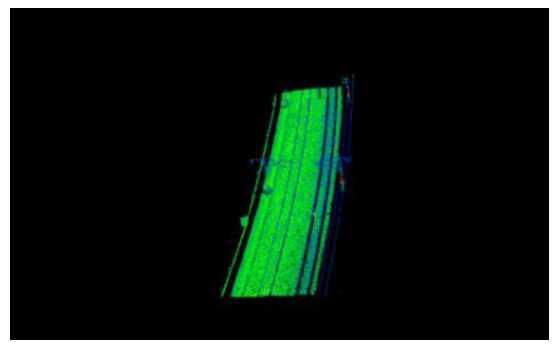

b. After preprocessing point cloud data

Figure 4. Pictures before and after preprocessing

\subsection{Rail Segmentation}

Point cloud segmentation divides point cloud into different partitions based on the spatial features, geometric features and texture features, which makes the point cloud in the same partition have similar features. Railway is a large-scale belt structure, and its point cloud data is large. It is necessary to segment the rail from the massive point cloud data at first. At present, the common point cloud segmentation methods include clustering segmentation, segmentation based on random sampling consistency and segmentation based on region growth [9-11]. This thesis proposed region growing segmentation based on the reflection intensity of point cloud, segmenting the rail according to the reflection characteristics, geometric dimensions and continuous characteristics of rail. The effect of segmentation is shown in Figure 5.

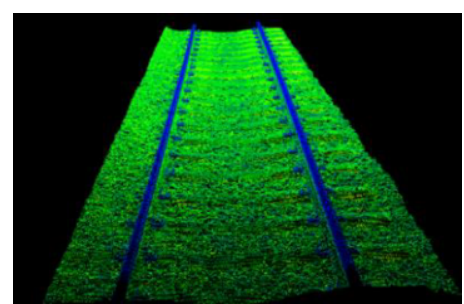

a. Before rail segmentation

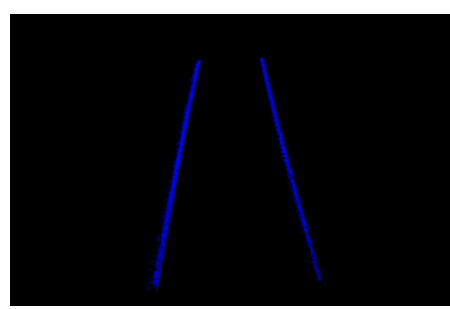

b. After rail segmentation

Figure 5. The effect of segmentation

The basic ideas of algorithm are as follows:

- According to the reflection characteristics of rail, the threshold $I$ of rail reflection intensity is set to filter out the points that do not match the reflection characteristics of rail, and the points that conform to the reflection characteristics of rail are saved. The selected unordered point cloud is sorted according to the railway mileage from small to large; 
- A point $P_{0}$ at the starting mileage of the target rail is designated as the seed point. According to the rail geometry size, the space distance threshold $R$ is set, and the points whose space distance from point $P_{0}$ is less than $R$ are selected as the rail partial points;

- $\quad$ Find the point $P_{l}$ among some rail points which is furthest from the point $P_{0}$, and select $P_{l}$ as seed point, $R$ as the distance threshold to gradually replace the seed points until all rail points are selected.

The algorithm only needs to specify seed points once each time to extract a single rail, and then does not need to segment the left and right rails, which greatly improves the efficiency of rail segmentation.

\subsection{Extraction of Rail Top Surface Point}

The experimental results show that the region growing segmentation algorithm based on reflection intensity has achieved good results in the segmentation of railway measurement. After the rail is segmented, the top surface points of rail need to be further extracted to calculate the track geometry. The top surface of rail can be regarded as an approximate horizontal plane, and the elevation of adjacent points is similar. Therefore, the top surface points of rail can be further extracted from the rail profile by using the standard deviation of elevation. Taking any point $P$ on the top surface of rail as an example, the elevation of many adjacent points in the field of point $P$ are $h_{1} 、 h_{2} 、 \ldots \ldots, h_{n}$, then the average elevation $\bar{h}$ :

$$
\bar{h}=\frac{1}{n} \sum_{i=1}^{n} h_{i}
$$

The elevation standard deviation of points in the field of point $P$ is:

$$
\sigma_{p}=\sqrt{\frac{1}{n} \sum_{i=1}^{n}\left(h_{i}-h\right)^{2}}
$$

Whether a point on the rail is a surface point is determined by the standard deviation of elevation. The larger the elevation standard deviation- $\sigma_{P}$ of the rail point $P$ is, the more obvious the change of the point elevation in the field of point $P$ is; otherwise, the smaller the elevation- $\sigma_{P}$ of the point $P$ in the point field is. The elevation standard deviation of the surface point is close to 0 , and the elevation standard deviation of the rail side point is large. Therefore, it can be separated from the rail point by using the characteristics of the elevation standard deviation of the top surface points of rail. As shown in Figure 6, the red point is the top point, and the green point is the rail waist point.

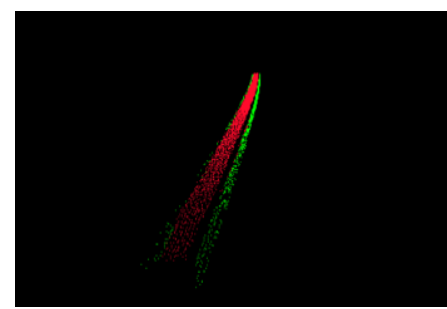

Figure 6. Extraction of top surface points of rail 


\section{Fitting of Plane Parameters of Railway Line}

\subsection{Rail Line Roughly Segment}

The surface points and the top surface points of rail are sequentially extracted from the massive raw point cloud data. Compared with the original data, the amount of point cloud data of the top surface points of rail is greatly reduced, but the amount of data of top surface points of rail in tens of kilometers is also very large. In order to reduce the amount of calculation, this thesis adopts the method based on the fixed-length chord gradient change rate of the rail line to reduce the sampling points. The fixed-length chord is the distance between two adjacent measuring points of the rail. The fixedlength chord needs to be selected when setting it, if the fixed length chord is set too much, the railway line segmentation accuracy will be reduced; if it is too small, the railway line segmentation will be unclear. In this paper, the chord length is set from $4.99 \mathrm{~m}$ to $5.01 \mathrm{~m}$ to screen the measure points of the right rail top surface [12]. The measure points distribution of the right rail after subsampling is shown in Figure 7.

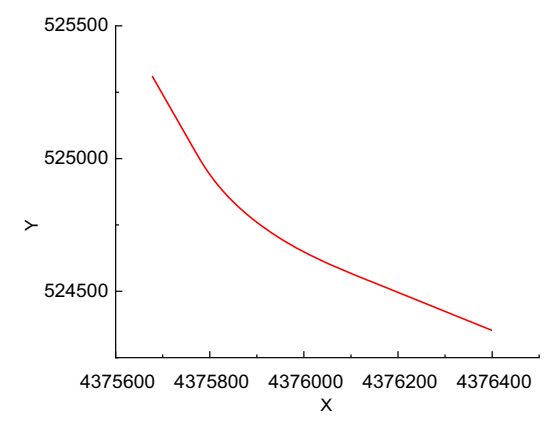

Figure 7. Measuring points distribution of the right rail

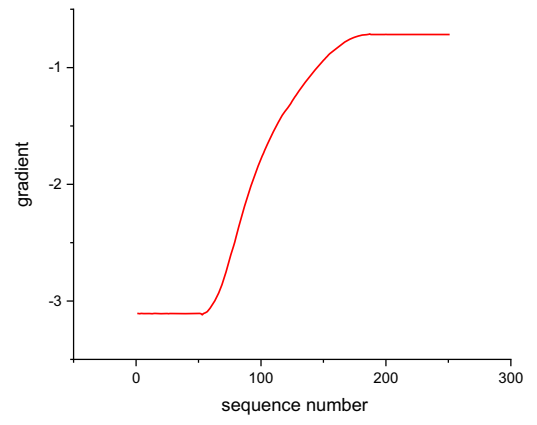

Figure 8. The sequence number-gradient diagram

The measuring points of the right rail consist of the measuring points of straight line segment, transition curve segment and circular curve segment. If the equation of straight-line segment and circular curve segment of rail line is fitted by the least orthogonal multiplication, the measuring points of the right rail must be roughly segmented. By calculating the gradient value of the measuring point- $i$ of the right rail and the measuring point $-i+1$ of the right rail as the gradient of the measuring point $-i$ of the right rail, the sequence number-gradient diagram is generated. As shown in Figure 8, the gradient value of the straight-line segment is basically a constant value. Select some measuring points of the straight-line segment and use the orthogonal least square algorithm to calculate the intersection point and rotation angle of the curve.

According to the principle that the point closest to the intersection point of the two adjacent straight lines must be the point on the circular curve, the point $P$ nearest to the intersection point is found out among all the measuring points. Select the measuring points at a certain distance from point $P$ as measuring points of circular curve segment, and then fit the curvilinear equation by the orthogonal least square algorithm.

\subsection{Straight-line Segment Fitting}

Straight-line segment fitting is to find the rules from the measuring points of the straight-line segment, and determine a straight line so that it can closely reflect the 
distribution of the measuring points on the straight-line segment. This straight line is used to represent the direction of the straight-line segment of the rail. The simplest line element in the railway is a straight line, and the straight line equation is:

$$
y_{i}=a x_{i}+b(i=1,2,3 \cdots n)
$$

In the above formula: $\left(x_{i}, y_{i}\right)$ is the coordinate of the measuring point of the right rail of the straight line.

$a, b$ are the gradient and intercept of the linear equation that will be solved later, set $a^{0}, b^{0}$ as their approximate values, $\delta a 、 \delta b$ as their correction numbers, the following formula can be obtained:

$$
\left\{\begin{array}{l}
a=a^{0}+\delta a \\
b=b^{0}+\delta b
\end{array}\right.
$$

The distance $S_{i}$ from any point $\left(x_{i}, y_{i}\right)$ to the straight line is:

$$
S_{i}=\sqrt{\frac{\left(a x_{i}-y_{i}+b\right)^{2}}{a^{2}+1}}
$$

The measurement points of each straight-line segment are calculated according to formula (5) and linearized with Taylor formula. The matrix form of error equation of all points can be expressed as follows:

$$
V=B \delta x-l
$$

In the formula (6), $V$ is the distance matrix between the measurement points of each straight-line segment and the fitting straight-line, $B$ is the unknown coefficient matrix after the linearization of formula (5), $\delta x$ is the correction matrix of slope intercept of linear equation, and $l$ is the constant term matrix after the linearization of formula (5).

$$
\begin{aligned}
& \text { Using } \sum_{i=1}^{n} S_{i}=\min \text { as the principle to obtain unknown parameters: } \\
& \delta x=\left[\begin{array}{c}
\delta a \\
\delta b
\end{array}\right]=\left(B^{T} B\right)^{-1}\left(B^{T} l\right)
\end{aligned}
$$

The correction $\delta a$ and $\delta b$ of slope intercept of linear equation is calculated by formula (7), and the linear equation is obtained by substituting the correction into Eq. (4). According to the principle of orthogonal least square algorithm, a program is written in VBA to find the line equation. 


\subsection{Circular Curve Fitting}

Two adjacent linear equations are fitted by the orthogonal least square algorithm, and the intersection point is the line intersection point. The part of points closest to the intersection point is taken as the points on the circular curve, and the partial points are used to fit the curvilinear equation through the orthogonal least square algorithm [13].

Suppose the curvilinear equation of railway is:

$$
\left(x_{i}-X\right)^{2}+\left(y_{i}-Y\right)^{2}=R^{2}(i=1,2,3 \cdots n)
$$

In formula (8), $\left(x_{i}, y_{i}\right)$ are the coordinates of the measuring point of the right rail of the circular curve, $X 、 Y 、 R$ are all parameters to be sought.

Select two endpoints and intermediate point from measuring points of circular curve segment, which are $\left(x_{1}, y_{1}\right) 、\left(x_{m}, y_{m}\right) 、\left(x_{n}, y_{n}\right)$. Calculate the approximate value of coordinates of circle center $\left(X^{0}, Y^{0}\right)$ and radius $R^{0}$ of the circular curve from the above three points. The formula (9) is obtained by considering the correction $\delta X, \delta Y$ and $\delta R$ of the center coordinate and radius of the circle.

$$
\left\{\begin{array}{l}
X=X^{0}+\delta X \\
Y=Y^{0}+\delta Y \\
R=R^{0}+\delta R
\end{array}\right.
$$

The distance from any measuring point $\left(x_{i}, y_{i}\right)$ of the circular curve segment to the circular curve equation is:

$$
D_{i}=\sqrt{\left(x_{i}-X\right)^{2}+\left(y_{i}-Y\right)^{2}}-R
$$

The measurement points of each circular curve segment are calculated according to formula (10) and linearized with Taylor formula to obtain the matrix form of error equation of all measuring points:

$$
V=B \delta x-l
$$

In the formula (11), $V$ is the distance matrix between the measuring point of each circular curve segment and the fitting circular curve equation, $B$ is the unknown coefficient matrix after the linearization of formula (8), $\delta x$ is the correction matrix of center coordinate and radius, and $l$ is the constant term matrix after the linearization of formula (8).

$$
\begin{gathered}
\text { Using } \sum_{i=1}^{n} D_{i}=\min \text { as the principle to obtain unknown parameters: } \\
\delta x=\left[\begin{array}{c}
\delta X \\
\delta Y \\
\delta R
\end{array}\right]=\left(B^{T} B\right)^{-1}\left(B^{T} l\right)
\end{gathered}
$$


The correction $\delta X, \delta Y$ and $\delta R$ of center coordinate and radius are calculated by formula (12). What's more, the optimal values of the center coordinates and radius of the circular curve could be obtained, and the optimal equation of the circular curve could be obtained.

\subsection{Calculation of Line Parameters}

After roughly segmenting, the orthogonal least square algorithm is used to fit the straight-line segment and curvilinear equation to obtain the corresponding transition curve parameters and calculate the plane coordinates of the line segment points. The parameters of straight-line, circular curve and transition curve of the track are obtained by iterative fitting until the plane distance between two adjacent segmented points is less than the tolerance [14-16]. Combined with the plane geometric relationship between the left and right tracks and the midline, the parameters of the straight line and circular curve of the midline are calculated, and then the curve elements of the midline are determined.

Table 1. The curve elements

\begin{tabular}{cccccc}
\hline & $\begin{array}{c}\text { Transition } \\
\text { curve }\end{array}$ & $\begin{array}{c}\text { Circular } \\
\text { curve }\end{array}$ & $\begin{array}{c}\text { Curve } \\
\text { radius }\end{array}$ & $\begin{array}{c}\text { Apex } \\
\text { distance }\end{array}$ & $\begin{array}{c}\text { Tangent } \\
\text { length }\end{array}$ \\
\hline $\begin{array}{c}\text { Fitting value of self-mobile } \\
\text { scanning measurement system }\end{array}$ & 149.162 & 659.621 & 800.077 & 43.731 & 339.197 \\
Fitting value of total station & 150.319 & 661.112 & 800.084 & 43.755 & 339.949 \\
Design value & 150 & 660.396 & 800 & 43.738 & 339.587 \\
\hline
\end{tabular}

From Table 1, combining the values of each curve element, the self-mobile scanning measurement system is closer to the design value than the traditional total station measurement, and the data obtained by the self-mobile scanning measurement system has continuity, which is more accurate than the discrete point data obtained by the traditional total station, and can better reflect the changes of track line. The curve radius and transition curve length fitted by the point cloud data collected from the selfmobile scanning measurement system are basically consistent with the design values. After calculation, the maximum deviation between the design and the actual measurement occurs at $\mathrm{K} 147+050.62 \mathrm{~m}$, and the deviation value is $4 \mathrm{~mm}$, which indicates that the overall condition of the line is good, and the design algorithm of line coordinate collection and processing based on continuous point cloud data is feasible.

\section{Conclusion}

Remeasuring railway is the guarantee for the safe and stable operation of trains. The self-mobile scanning measurement system can replace the manual measurement to collect field data. This kind of data collection is safe, efficient and low-cost, which can make up for many shortcomings of the traditional measurement. The continuous spatial position information and panoramic image data of railway can be obtained. The obtained image data and point cloud data can be used in the 3D modeling of railway, line alignment parameter fitting, curve rectification, mileage measurement, detection of foreign body invasion and other projects, which is conducive to the establishment of railway database and the development of smart railway. The experimental results show that the proposed region growing segmentation based on the reflection intensity of 
point cloud has high efficiency and good stability. The alignment fitting and line parameters of railways can be automatically calculated by the CAD menu designed in this thesis, which will change the current situation of railway measurement to a certain extent, and greatly improve the efficiency of remeasuring railways.

\section{Acknowledgement}

My deepest gratitude goes first and foremost to my tutor, for his constant encouragement and guidance. He has walked me through all the stages of the writing of the thesis. Secondly, I would like to express my heartfelt gratitude to his National Natural Science Foundation of China (51568037). In addition, I deeply appreciate the contribution of lzjtu (201802) EP.

\section{References}

[1] Han F. Research on status setection and evaluation technology of existing railway based on point cloud information. Wuhan University Press, 2016.

[2] Wang XK. Research and application of railway robot inspection system safety. Railway Engineering, 2013(02): 81-83.

[3] Chen X, Ao Q, Xu LL. Research analysis and accuracy evaluation of mobile surveying system (Multiplatform LiDAR System). Geomatics \& Spatial Information Technology, 2020, 43(S1): 84-87+90.

[4] Chen ZY. Research and application of railway robot inspection system safety. Railway Standard Design, 2020(65): 1-7.

[5] Tang JF. Research on the technology of existing railway lines retesting based on vehicle laser radar measurement. Journal of Railway Engineering Society, 2016, 33(12): 43-47.

[6] Duan XF, Han F, Li LY, Wei P. Research on rail track point cloud data visualization design reconstruction method. Railway Standard Design, 2017, 61(04): 14-18.

[7] Zhang B, Xiong CB. Automatic point cloud registration based on voxel down sampling and key point extraction. Laser \& Optoelectronics Progress, 2020, 57(04): 109-117.

[8] Lu DD, Zou JG. Comparative research on denoising algorithms of 3D laser point cloud[J]. Bulletin of Surveying and Mapping, 2019(S2): 102-105.

[9] Cheng YJ, Qiu WG, Duan DY. Automatic creation of as-is building information model from singletrack railway tunnel point clouds. Automation in Construction, 2019, 106.

[10] Huang LL, Li WG, Yang QL, Chen YC. Segmentation algorithm of three-dimensional point cloud data based on region growing. Applied Mechanics and Materials, 2015(741): 382-385.

[11] Zhang S, Wang C, Yang Z, et al. Automatic railway power line extraction using mobile laser scanning data. Isprs International Archives of the Photogrammetry Remote Sensing \& Spatial Information Sciences, 2016, XLI-B5: 615-619.

[12] Liu XY. Research on extraction of retest elements of existing railway based on vehicle LiDAR Technology. Chang'an University, 2018.

[13] Ding KL, Ou JK, Zhao CM. Orthogonal least squares curve fitting method. Science of Surveying and Mapping, 2007(03): 18-19+17+192.

[14] Pan ZX, Han F, Wang B, Niu JD, Zhang SJ. Design and practice of existing railway line realignment algorithm based on curvature variation characteristics. Railway Standard Design, 2019, 63(09): 6-10.

[15] Li HY, Chen ZY, Xing C, Chen DS, Chai DM. Calculation method for curve re-surveying of the existing railway line. China Railway Science, 2009, 30(02): 18-22.

[16] Zhao CS, Han F. The unified calculating model of middle stake coordinates in railway. Journal of Lanzhou Jiaotong University, 2009, 28(01): 49-51. 